\title{
Aspirin attenuates angiotensin II-induced inflammation in bone marrow mesenchymal stem cells via the inhibition of ERK1/2 and NF-KB activation
}

\author{
FENXI ZHANG, MING LU, HUAIBIN WANG and TONGMING REN \\ Department of Anatomy, Sanquan College, Xinxiang Medical University, Xinxiang, Henan 453003, P.R. China
}

Received July 12, 2013; Accepted August 19, 2013

DOI: $10.3892 /$ br.2013.160

\begin{abstract}
Angiotensin II (Ang II) is a peptide hormone that plays a critical role in numerous physiological and pathophysiological processes. It is also commonly used as an inducer for the directional differentiation of bone marrow mesenchymal stem cells (bmMSCs). Previous studies demonstrated that Ang II induces inflammatory responses in endothelial cells, smooth muscle cells and fibroblasts. Aspirin is generally used as analgesic, antipyretic and occasionally anti-inflammatory medication. Whether aspirin suppresses inflammatory responses in bmMSCs has not been elucidated. In this study, we investigated the effect of aspirin on Ang II-induced inflammation in bmMSCs. Our results demonstrated that Ang II $(10 \mathrm{nM}-10 \mu \mathrm{M})$ increased the secretion of tumor necrosis factor (TNF)- $\alpha$ and interleukin (IL)- 6 from bmMSCs in a dose-dependent manner. This result was further confirmed by a reverse transcription-polymerase chain reaction (RT-PCR) assay, which demonstrated a dose-dependent increase in the mRNA expression of TNF- $\alpha$, IL- 6 , IL-1 $\beta$ and monocyte chemotactic protein-1 (MCP-1) in bmMSCs following exposure to Ang II. Furthermore, it was also observed that Ang II increased the expression of phospho-extracellular signal-regulated kinase 1/2 (ERK1/2) and phospho-nuclear factor $\kappa$-light-chain-enhancer of activated B cells (NF- $\kappa \mathrm{B})-\mathrm{p} 65$ in bmMSCs. The application of aspirin $(0.1 \mathrm{mM})$ significantly inhibited the activation of ERK1/2 and $\mathrm{NF}-\kappa \mathrm{B}$, the expression of TNF- $\alpha$, IL-6, IL-1 $\beta$ and MCP-1 genes and the secretion of TNF- $\alpha$ and IL-6. Our findings indicated that aspirin may attenuate Ang II-induced inflammation in bmMSCs via the inhibition of ERK1/2 and $\mathrm{NF}-\kappa \mathrm{B}$ activation.
\end{abstract}

Correspondence to: Dr Fenxi Zhang, Department of Anatomy, Sanquan College, Xinxiang Medical University, 601 Jinshui Road, Xinxiang, Henan 453003, P.R. China

E-mail: fxzhang0824@gmail.com

Key words: aspirin, angiotensin II, bone marrow mesenchymal stem cells, inflammation, extracellular signal-regulated kinase 1/2, nuclear factor $\kappa$-light-chain-enhancer of activated B cells

\section{Introduction}

Bone marrow mesenchymal stem cells (bmMSCs) are a type of multipotent stem cells that are derived from the adult bone marrow and are able to differentiate into a variety of cell types, such as osteoblasts, chondrocytes, adipocytes and cardiomyocytes $(1,2)$. Angiotensin II (Ang II) is a peptide hormone that plays a critical role in a series of physiological and pathophysiological processes, including cell proliferation, differentiation, apoptosis and inflammation (3). Recently, Ang II was used to induce the differentiation of bmMSCs to other functional cell lineages, such as cardiomyocytes, adipocytes and smooth muscle-like cells (4-6). It was demonstrated that Ang II induces inflammatory responses in vitro in several types of cells, including endothelial cells, smooth muscle cells, fibroblasts and kidney tubule epithelial cells (7-9).

Aspirin is a drug commonly used as analgesic, antipyretic and occasionally anti-inflammatory medication (8). Recent studies demonstrated that aspirin may suppress inflammatory responses in cultured endothelial cells, fibroblasts and other cell lines, via the inhibition of reactive oxygen species (ROS) generation $(8,10,11)$. Ang II, as a strong inducer of ROS generation, may induce inflammatory responses in bmMSCs and aspirin may attenuate these inflammatory responses. The purpose of the present study was to investigate the effects of aspirin on Ang II-induced inflammation in bmMSCs and the possible underlying mechanisms.

\section{Materials and methods}

Materials and reagents. Aspirin, Ang II and $2 \mathrm{X}$ PCR Reaction mix were purchased from Sigma-Aldrich (St. Louis, MO, USA). The mouse tumor necrosis factor $\alpha$ (TNF- $\alpha)$ Quantikine ELISA kit and the mouse interleukin (IL)-6 Quantikine ELISA kit were purchased from R\&D Systems Inc. (Minneapolis, MN, USA). DNase I, RNeasy Mini kit and SuperScript II First-Strand cDNA Synthesis kit were purchased from Invitrogen Life Technologies (Carlsbad, CA, USA). Rabbit anti-mouse phospho-extracellular signal-regulated protein 1/2 (ERK1/2), ERK1/2, phospho-nuclear factor $\kappa$-light-chain-enhancer of activated $B$ cells (NF- $\mathrm{B})-\mathrm{p} 65$ and

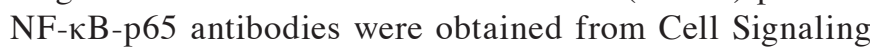
Technology, Inc. (Danvers, MA, USA). $\beta$-actin antibody and 
horseradish peroxidase (HRP)-conjugated goat anti-rabbit secondary antibody were purchased from Abcam (Cambridge, MA, USA). ECL Western Blotting Substrate was purchased from Thermo Scientific (Rockford, IL, USA). The polyvinylidene fluoride (PVDF) membranes were obtained from GE Healthcare (Pittsburgh, PA, USA).

Cell culture and study protocol. BmMSCs were obtained as previously described $(12,13)$. In brief, bone marrow was harvested from the mouse tibia and femur, washed and cultured in Dulbecco's modified Eagle's medium supplemented with $15 \%$ fetal bovine serum for $3 \mathrm{~h}$. Subsequently, the non-adherent cells were removed and the medium was replaced. A purified population of bmMSCs was obtained after 3 weeks of culture. The cells were plated in 6- and 12-well plates and treated with $0,10 \mathrm{nM}, 100 \mathrm{nM}, 1 \mu \mathrm{M}$ and $10 \mu \mathrm{M}$ Ang II for $12 \mathrm{~h}$. In other experiments, the cells were pretreated with $0.1 \mathrm{mM}$ aspirin for $30 \mathrm{~min}$ and then exposed to $1 \mu \mathrm{M}$ Ang II for an additional $12 \mathrm{~h}$.

Enzyme-linked immunosorbent assay (ELISA). Following treatment with Ang II and aspirin, the supernatants of the growth medium were collected by centrifugation and frozen at $-80^{\circ} \mathrm{C}$ until use. The levels of TNF- $\alpha$ and IL- 6 were measured using the mouse TNF- $\alpha$ Quantikine ELISA kit and the mouse IL-6 Quantikine ELISA kit, according to the manufacturer's instructions. Absorbance at $450 \mathrm{~nm}$ was read by a microplate reader.

Western blot assay. Proteins were extracted from the treated bmMSCs and separated by $12 \%$ sodium dodecyl sulphate-polyacrylamide gel electrophoresis. Following electrophoresis, the proteins were transferred to the PVDF membranes. The membranes were blocked with $5 \%$ bovine serum albumin in Tris-buffered saline with Tween-20 (TBS-T) and then incubated with phospho-ERK1/2, ERK1/2, phospho-NF- $\kappa$ B-p65, NF- $\kappa$ B-p65 and $\beta$-actin antibodies at $4{ }^{\circ} \mathrm{C}$ overnight. Subsequently, the blots were washed with TBS-T and incubated with HRP-conjugated secondary antibody for $1 \mathrm{~h}$ at room temperature. The immunoreactive bands were visualized by enhanced chemiluminescence.

Reverse transcription-polymerase chain reaction (RT-PCR) assay. Total RNA was extracted from the treated bmMSCs with a RNeasy Mini kit and complementary DNA (cDNA) was synthesized with a SuperScript II First-Strand cDNA Synthesis kit. To eliminate contamination of the genomic DNA, RNA was pretreated with DNase I prior to the synthesis of cDNA. RT-PCR was performed using $2 \mathrm{X}$ PCR reaction solution with $100 \mathrm{ng}$ cDNA and $0.3 \mu \mathrm{M}$ primers. The primer sequences and reaction cycles are listed in Table I.

Statistical analysis. Statistical analysis was performed with SPSS software, version 11.5 (SPSS Inc., Chicago, IL, USA). Data are presented as means \pm standard deviation (SD) from 4 independent experiments. Univariate comparisons of the means were evaluated using the Student's t-test and/or one-way analysis of variance with Tukey's post hoc adjustment for multiple comparisons when appropriate. $\mathrm{P}<0.05$ was considered to indicate a statistically significant difference.

\section{Results}

Ang II induces inflammation in bmMSCs. In this study, the levels of TNF- $\alpha$ and IL- 6 were evaluated by ELISA. As shown in Fig. 1A and B, TNF- $\alpha$ and IL- 6 were increased in a dose-dependent manner in the growth medium with the cultured bmMSCs as the cells were exposed to $10 \mathrm{nM}-10 \mu \mathrm{M}$ Ang II. These data were further confirmed by an RT-PCR assay, which demonstrated a dose-dependent increase in TNF- $\alpha$ and IL-6 mRNA in the bmMSCs following exposure to $10 \mathrm{nM}-10 \mu \mathrm{M}$ Ang II (Fig. 1C,D and F). In addition, a significant increase in IL-1 $\beta$ and monocyte chemotactic protein-1 (MCP-1) mRNA was also observed in the bmMSCs following exposure to different concentrations of Ang II (Fig. 1C,E and G).

Ang II activates ERKI/2 and NF- $\kappa B$ signals in bmMSCs. Western blotting demonstrated that treatment with Ang II $(10 \mathrm{nM}-10 \mu \mathrm{M})$ increased the expression of phospho-ERK1/2 and phospho-NF- $\mathrm{B}-\mathrm{p} 65$ in bmMSCs in a dose-dependent manner; however, Ang II did not significantly affect the total expression of ERK1/2 and NF- $\mathrm{B}-\mathrm{p} 65$ (Fig. 1H-L). These data demonstrated that ERK $1 / 2$ and $N F-\kappa B$ are activated in bmMSCs following exposure to different concentrations of Ang II.

Aspirin inhibits the activation of ERK1/2 and $N F-\kappa B$. Based on preliminary data, $1 \mu \mathrm{M}$ Ang II was used in the following experiments to investigate the effect of aspirin on Ang II-induced inflammation. As shown in Fig. 2A-E, the application of aspirin $(0.1 \mathrm{mM})$ significantly inhibited the Ang II-induced expression of phospho-ERK1/2 and phospho-NF- $\mathrm{B}-\mathrm{p} 65$, although it did not significantly affect the total expression of ERK1/2 and NF- $\kappa$ B-p65.

Aspirin suppresses Ang II-induced inflammation. The RT-PCR assay demonstrated that the application of aspirin significantly inhibited the Ang II-induced expression of TNF- $\alpha$, MCP-1, IL- 6 and IL-1 $\beta$ genes (Fig. 2F-J). In addition, the ELISA revealed that aspirin $(0.1 \mathrm{mM})$ significantly inhibited the Ang II-induced secretion of TNF- $\alpha$ and IL-6 from bmMSCs (Fig. 2K and L).

\section{Discussion}

The present study demonstrated that Ang II induces inflammatory responses and the activation of ERK $1 / 2$ and $\mathrm{NF}-\kappa \mathrm{B}$ in bmMSCs and that the application of aspirin inhibits the Ang II-induced activation of ERK1/2 and NF- $\kappa \mathrm{B}$ and inflammatory responses, indicating that aspirin may inhibit Ang II-induced inflammation in bmMSCs via the inhibition of ERK1/2 and NF- $\kappa$ B activation.

BmMSCs are the most promising source of stem cells for cell transplantation therapy and have been widely used in tissue regenerative medicine. Generally, bmMSCs must be induced in vitro by chemical or biological reagents for directional differentiation prior to transplantation. Ang II is one of the most commonly used inducers of MSC differentiation. It was demonstrated that Ang II is able to induce the differentiation of bmMSCs to cardiomyocytes, adipocytes and smooth 
Table I. Primers for reverse transcription-polymerase chain reaction.

\begin{tabular}{llcc}
\hline Primer & \multicolumn{1}{c}{ Sequence } & Product size (bp) & Reaction cycles \\
\hline TNF- $\alpha$ & $\begin{array}{l}\text { Sense: 5'-CCGATGGGTTGTACCTTGTC-3' } \\
\text { Antisense: 5'-GGGCTGGGTAGAGAATGGAT-3' }\end{array}$ & 352 & 32 \\
MCP-1 & $\begin{array}{l}\text { Sense 5'-GGAGCATCCACGTGTTGGC-'3 } \\
\text { Antisense: 5'-GTAGGAGTGACCAGTGTGACAGT-3' }\end{array}$ & 391 & 33 \\
IL-6 & Sense: 5'-GATGCTACCAAACTGGATATAATC-3' & 269 & 37 \\
& Antisense: 5'-GGTCCTTAGCCACTCCTTCTGTG-3' & & 38 \\
IL-1 $\beta$ & Sense: 5'-GAAATGCCACCTTTTGACAGTG-3' & 225 & 32 \\
$\beta$-actin & $\begin{array}{l}\text { Antisense: 5'-GAAGGTCCACGGGAAAGACAC-3' } \\
\text { Sense: 5'-TTCTTTGCAGCTCCTTCGTTGCCG-3' }\end{array}$ & 458 & \\
& Antisense: 5'-TGGATGGCTACGTACATGGCTGGG-3' & &
\end{tabular}

TNF- $\alpha$, tumor necrosis factor $\alpha$; MCP-1, monocyte chemotactic protein-1; IL, interleukin.
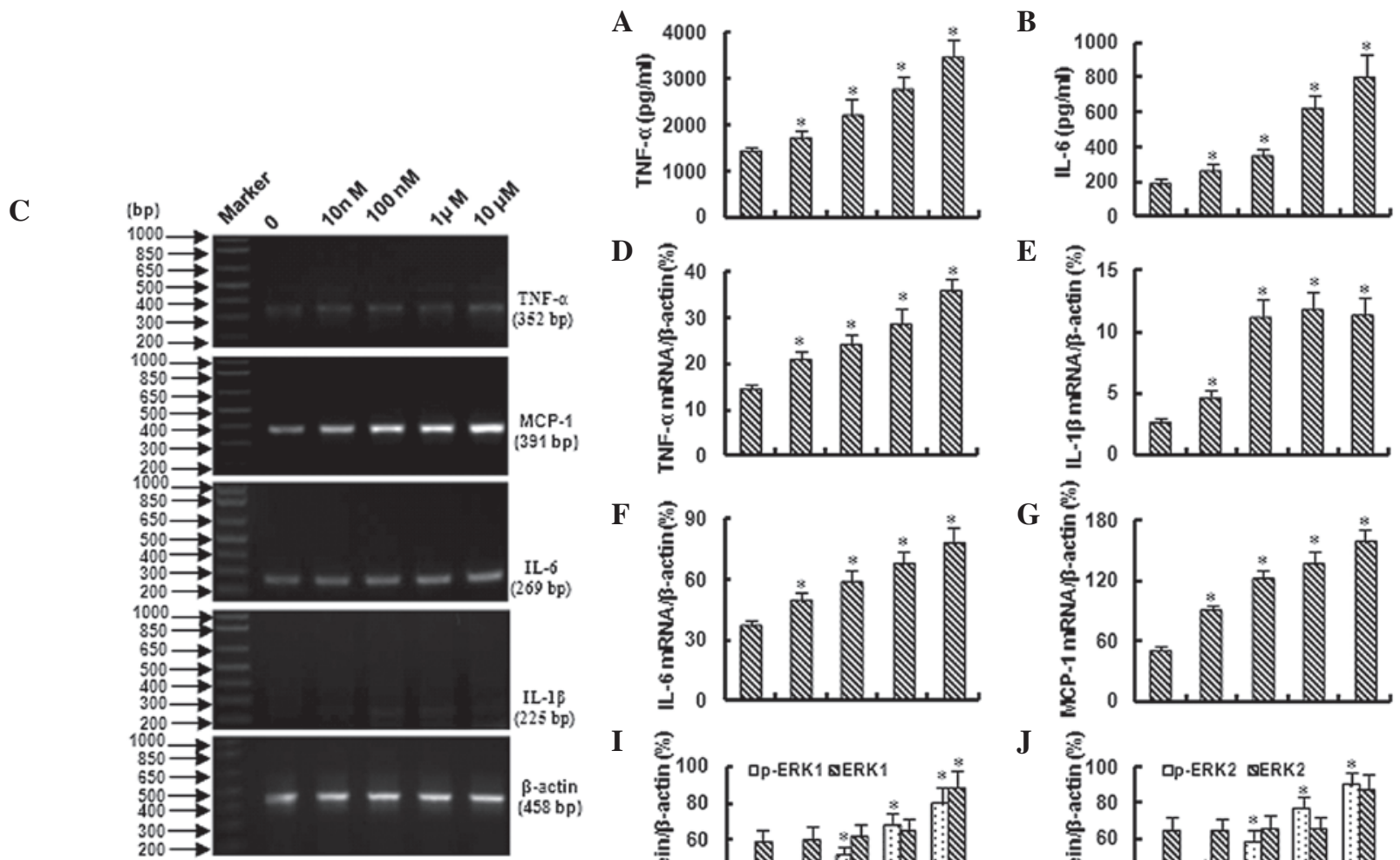

E
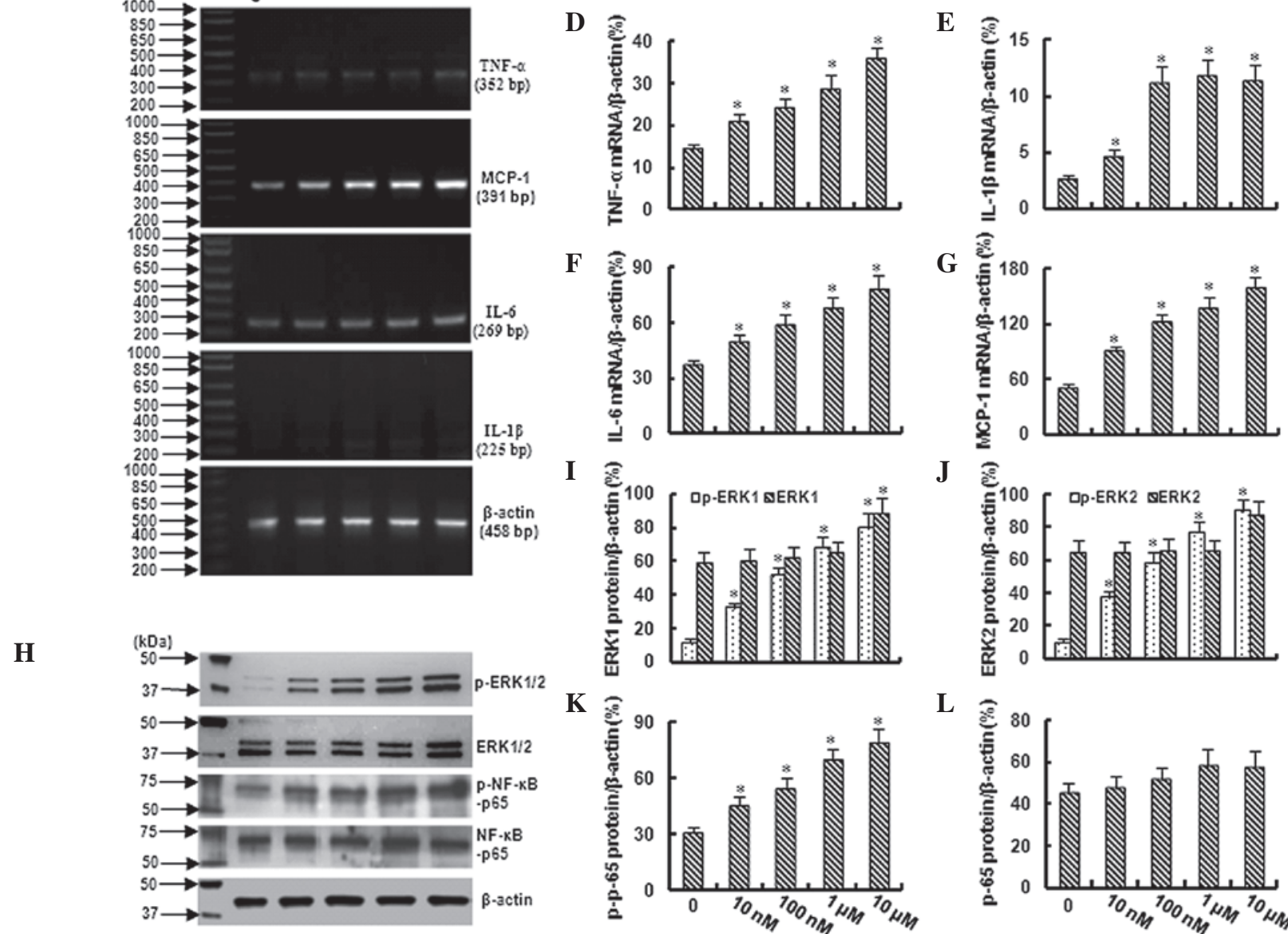

Figure 1. Angiotensin II (Ang II) induces inflammation in bone marrow mesenchymal stem cells (bmMSCs). (A,B) The ELISA demonstrated the levels of tumor necrosis factor $\alpha$ (TNF- $\alpha$ ) and interleukin (IL)-6 in the medium with the cultured bmMSCs. (C-G) The reverse transcription-polymerase chain reaction assay demonstrated the expression of TNF- $\alpha$, IL-6, IL-1 $\beta$ and monocyte chemotactic protein-1 (MCP-1) in bmMSCs exposed to $10 \mathrm{nM}-10 \mu \mathrm{M}$ Ang II for $12 \mathrm{~h}$. (H-L) Western blotting demonstrated the expression of extracellular signal-regulated protein-1/-2 (ERK1/2), nuclear factor $\kappa$-light-chain-enhancer of activated

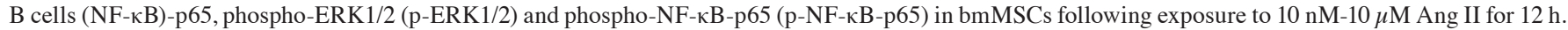
The bar graphs represent means $\pm \mathrm{SD}\left(4\right.$ independent experiments/group). ${ }^{*} \mathrm{P}<0.05$ vs. control. 
A

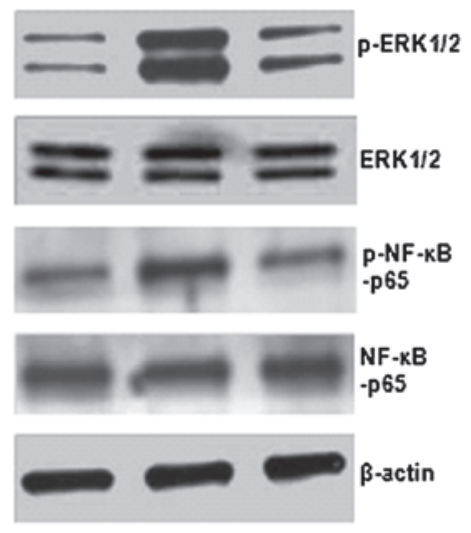

F
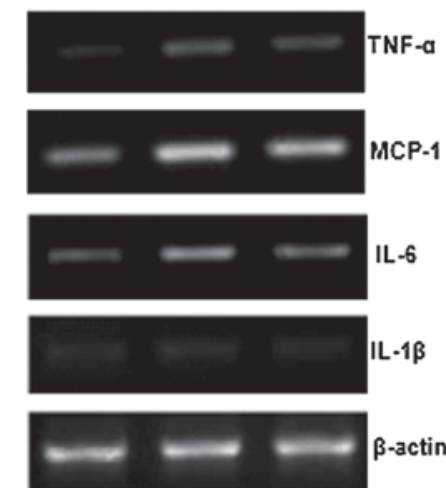

$\begin{array}{lll}\text { Angll } & - & +\end{array}+$

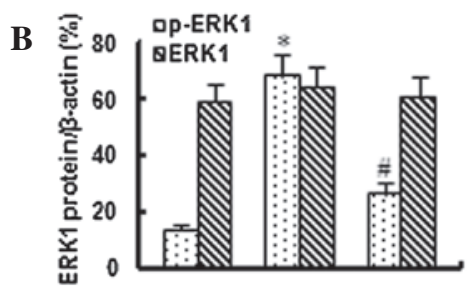

C

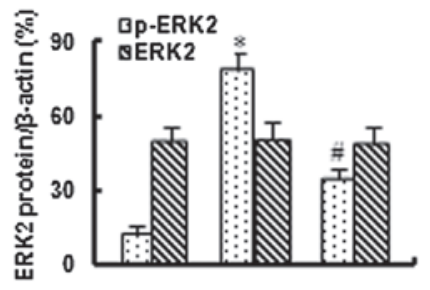

D

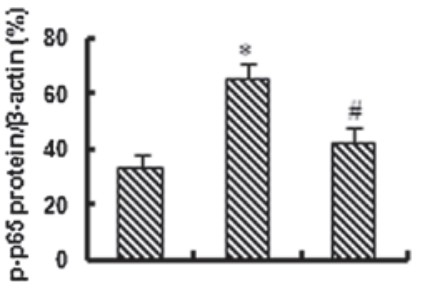

E

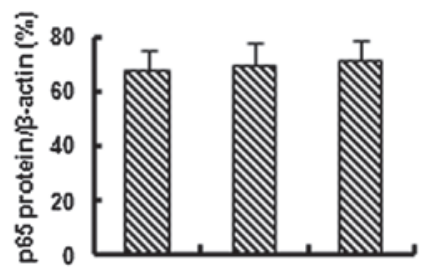

G

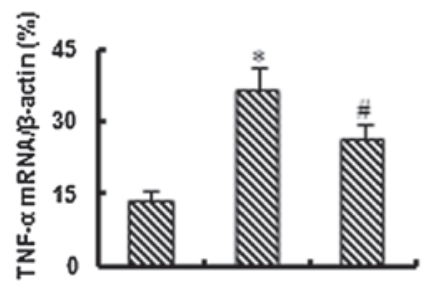

I

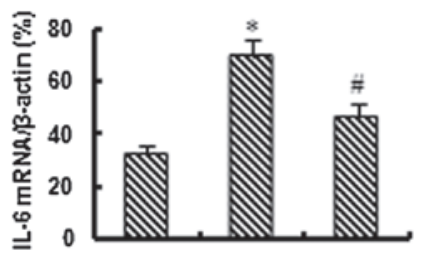

$\mathbf{H}$

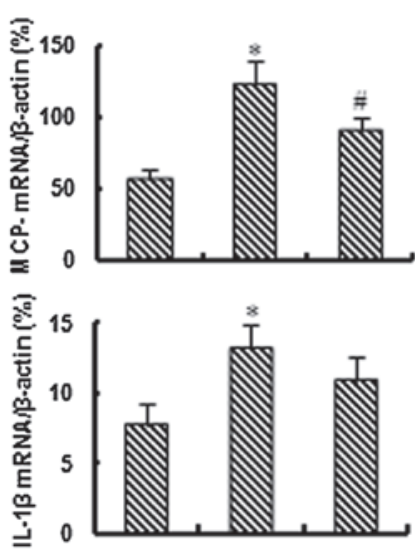

$\mathbf{L}$

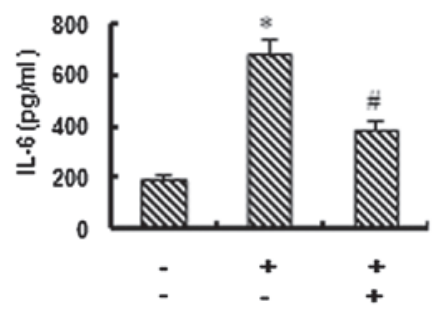

Figure 2. Aspirin inhibits angiotensin II (Ang II)-induced inflammation in bone marrow mesenchymal stem cells (bmMSCs). (A-E) Western blotting demonstrated that aspirin $(0.1 \mathrm{mM})$ inhibits the Ang II $(1 \mu \mathrm{M})$-induced expression of phospho-extracellular signal-regulated protein-1/-2 (p-ERK1/2) and phospho-nuclear factor $\kappa$-light-chain-enhancer of activated B cells (p-NF- $\kappa$ B) in bmMSCs. (F-J) The reverse transcription-polymerase chain reaction assay demonstrated that aspirin inhibits the Ang II-induced expression of tumor necrosis factor $\alpha$ (TNF- $\alpha$ ), interleukin (IL)-6, IL-1 $\beta$ and monocyte chemotactic protein-1 (MCP-1) in bmMSCs. (K,L) The ELISA demonstrated that aspirin inhibits the Ang II-induced secretion of TNF- $\alpha$ and IL-6 from bmMSCs. The bar graphs represent means $\pm \mathrm{SD}\left(4\right.$ independent experiments/group). ${ }^{*} \mathrm{P}<0.05$ vs. control and ${ }^{\#} \mathrm{P}<0.05$ vs. Ang II.

muscle cells (4-6). The present study demonstrated that treatment with Ang II may also induce inflammatory responses in bmMSCs.

It is known that the inflammatory responses in bmMSCs limit their clinical use. Treatment with anti-inflammatory and anti-immune rejection drugs may promote the survival of bmMSCs in recipient organs following transplantation (14). Previous studies demonstrated that aspirin inhibits the inflammatory responses in several cell lines, including endothelial cells and fibroblasts, via the inhibition of NADPH oxidase activity and ROS generation $(8,10)$. In the present study, we also observed that aspirin inhibited Ang II-induced inflammatory responses in bmMSCs.

ERK1/2 are members of the mitogen-activated protein kinase superfamily that is implicated in the development of acute and chronic inflammatory disorders $(15,16)$. It was reported that aspirin inhibits the activation of ERK1/2 and inflammatory responses in a series of pathophysiological conditions $(17,18)$. In the present study, we observed that aspirin $(0.1 \mathrm{mM})$ significantly inhibited the AngII-induced activation of ERK1/2 in bmMSCs. In addition, we also observed that aspirin markedly inhibited the Ang II-induced activation of $N F-\kappa B$. NF- $\kappa B$ is another important factor involved in inflammation (19). Previous studies demonstrated that aspirin inhibits the activation of $\mathrm{NF}-\kappa \mathrm{B}$ pathway in certain chronic inflammatory conditions, protecting organs and tissues from inflammation and damage (20-22). In this study, the inhibitory effects of aspirin on the Ang II-induced inflammation in bmMSCs may be attributed to its role in the regulation of the ERK1/2 and NF- $\kappa \mathrm{B}$ activity. 
In summary, we demonstrated that Ang II induces inflammatory responses and activation of ERK1/2 and NF- $\mathrm{KB}$ in bmMSCs in a dose-dependent manner and that the application of aspirin inhibits the Ang II-induced activation of ERK1/2, $\mathrm{NF}-\mathrm{\kappa B}$ and inflammatory responses. These findings suggest that aspirin may be effective in attenuating Ang II-induced inflammation in cultured bmMSCs and may serve as an alternative drug to suppress inflammatory responses when the cells are exposed to Ang II for directional differentiation.

\section{References}

1. Rostovskaya M and Anastassiadis K: Differential expression of surface markers in mouse bone marrow mesenchymal stromal cell subpopulations with distinct lineage commitment. PLoS One 7: e51221, 2012.

2. Jackson L, Jones DR, Scotting P, et al: Adult mesenchymal stem cells: differentiation potential and therapeutic applications. J Postgrad Med 53: 121-127, 2007.

3. Mehta PK and Griendling KK: Angiotensin II cell signaling: physiological and pathological effects in the cardiovascular system. Am J Physiol Cell Physiol 292: C82-C97, 2007.

4. Xing Y, Lv A, Wang L, et al: The combination of angiotensin II and 5-azacytidine promotes cardiomyocyte differentiation of rat bone marrow mesenchymal stem cells. Mol Cell Biochem 360: 279-287, 2012

5. Matsushita K, Wu Y, Okamoto Y, et al: Local renin angiotensin expression regulates human mesenchymal stem cell differentiation to adipocytes. Hypertension 48: 1095-1102, 2006.

6. Kim YM, Jeon ES, Kim MR, et al: Angiotensin II-induced differentiation of adipose tissue-derived mesenchymal stem cells to smooth muscle-like cells. Int J Biochem Cell Biol 40: 2482-2491, 2008.

7. Zhan Y, Brown C, Maynard E, et al: Ets-1 is a critical regulator of Ang II-mediated vascular inflammation and remodeling. J Clin Invest 115: 2508-2516, 2005.

8. Wang X, Lu J, Khaidakov M, et al: Aspirin suppresses cardiac fibroblast proliferation and collagen formation through downregulation of angiotensin type 1 receptor transcription. Toxicol Appl Pharmacol 259: 346-354, 2012.

9. Wolak T, Kim H, Ren Y, et al: Osteopontin modulates angiotensin II-induced inflammation, oxidative stress, and fibrosis of the kidney. Kidney Int 76: 32-43, 2009.
10. Chen JW, Zhou SB and Tan ZM: Aspirin and pravastatin reduce lectin-like oxidized low density lipoprotein receptor-1 expression, adhesion molecules and oxidative stress in human coronary artery endothelial cells. Chin Med J (Engl) 123: 1553-1556, 2010.

11. Wu Y, Zhai H, Wang Y, et al: Aspirin-triggered lipoxin $\mathrm{A}_{4}$ attenuates lipopolysaccharide-induced intracellular ROS in BV2 microglia cells by inhibiting the function of NADPH oxidase. Neurochem Res 37: 1690-1696, 2012.

12. Zhang F, Wang C, Jing S, et al: Lectin-like oxidized LDL receptor-1 expresses in mouse bone marrow-derived mesenchymal stem cells and stimulates their proliferation. Exp Cell Res 319: 1054-1059, 2013.

13. Zhang F, Jing S, Ren T, et al: microRNA-10b promotes the migration of mouse bone marrow-derived mesenchymal stem cells and downregulates the expression of E-cadherin. Mol Med Rep: Aug 6, 2013 (Epub ahead of print). doi: 10.3892/mmr.2013.1615.

14. van Velthoven CT, Kavelaars A and Heijnen CJ: Mesenchymal stem cells as a treatment for neonatal ischemic brain damage. Pediatr Res 71: 474-481, 2012.

15. Pastore S, Mascia F, Mariotti F, et al: ERK1/2 regulates epidermal chemokine expression and skin inflammation. J Immunol 174: 5047-5056, 2005.

16. Lee WK, Chung KW, Kim GH, et al: Gallotannin causes differentiation and inflammation via ERK-1/-2 and p38 kinase pathways in rabbit articular chondrocytes. Mol Med Rep 7: 701-707, 2013.

17. Vartiainen N, Goldsteins G, Keksa-Goldsteine V, et al: Aspirin inhibits p44/42 mitogen-activated protein kinase and is protective against hypoxia/reoxygenation neuronal damage. Stroke 34: 752-757, 2003.

18. Wang YP, Wu Y, Li LY, et al: Aspirin-triggered lipoxin $\mathrm{A}_{4}$ attenuates LPS-induced pro-inflammatory responses by inhibiting activation of NF- $\mathrm{KB}$ and MAPKs in BV-2 microglial cells. J Neuroinflammation 8: 95, 2011.

19. Ke X, Chen J, Zhang X, et al: Qing Hua Chang Yin attenuates lipopolysaccharide-induced inflammatory response in human intestinal cells by inhibiting NF- $\mathrm{B}$ activation. Exp Ther Med 6: 189-193, 2013.

20. Yamamoto Y and Gaynor RB: Therapeutic potential of inhibition of the NF-kappaB pathway in the treatment of inflammation and cancer. J Clin Invest 107: 135-142, 2001.

21. Muller DN, Heissmeyer V, Dechend R, et al: Aspirin inhibits NF-kappaB and protects from angiotensin II-induced organ damage. FASEB J 15: 1822-1824, 2001.

22. Akyazi I, Eraslan E, Gulcubuk A, et al: Long-term aspirin pretreatment in the prevention of cerulein-induced acute pancreatitis in rats. World J Gastroenterol 19: 2894-2903, 2013. 